\title{
Carotid-femoral pulse wave velocity is negatively correlated with aortic diameter
}

\author{
Marc A Bailey ${ }^{1,2,4}$, Jennifer M Davies ${ }^{1,4}$, Kathryn J Griffin ${ }^{1,2}$, Katherine I Bridge ${ }^{1,2}$, Anne B Johnson ${ }^{1}$, \\ Soroush Sohrabi ${ }^{1}$, Paul D Baxter ${ }^{3}$ and D Julian A Scott ${ }^{1,2}$
}

Cardiovascular events pose significant morbidity and mortality burden to abdominal aortic aneurysm (AAA) patients. Arterial stiffness as measured by pulse wave velocity (PWV) is an independent predictor of cardiovascular risk. We investigated the relationship between aortic diameter and PWV. Consecutive patients with AAA were invited to participate. Patients completed a health questionnaire, received aortic ultrasound and carotid-femoral PWV (cfPWV) recordings with a Vicorder. Thirty patients were used for reproducibility assessment. A linear regression model was used to identify significant predictors of cfPWV. Observer variation was assessed using Bland and Altman analysis and the intraclass correlation coefficient. Three hundred and nine patients were included-148 with AAA and 161 controls. The mean difference for repeated cfPWV between observers was $0.11 \mathrm{~ms}^{-1}$. cfPWV was positively correlated with age $(r=0.24, P<0.001)$ and systolic blood pressure $(r=0.29, P<0.001)$ and negatively correlated with aortic diameter $(r=-0.15, P=0.008)$. There was no difference in cfPWV between AAA and control groups $\left(9.75 \pm 2.3 \mathrm{~ms}^{-1}\right.$ vs. $\left.9.55 \pm 2.3 \mathrm{~ms}^{-1}, P=0.43\right)$. Aortic diameter $(P=0.003)$ and systolic blood pressure $(P<0.001)$ were significant predictors of cfPWV independent of age, aspirin usage and a history of myocardial infarction. Patients with large AAA $(>5 \mathrm{~cm})$ had decreased cfPWV compared with patients with small AAA $(P=0.02)$ or normal diameter aorta $(P=0.02)$. Vicorder measurements of cfPWV are repeatable. cfPWV is negatively associated with infra-renal aortic diameter and reduced in large AAA. cfPWV is likely invalid for accurate arterial stiffness assessment in patients with AAA owing to the apparent confounding effect of aortic size.

Hypertension Research (2014) 37, 926-932; doi:10.1038/hr.2014.101; published online 12 June 2014

Keywords: abdominal aortic aneurysm; aortic diameter; arterial stiffness; pulse wave velocity

\section{INTRODUCTION}

Abdominal aortic aneurysm (AAA) is a focal dilatation of the abdominal aorta, which poses a significant risk to life through rupture. Despite technological advances in the minimally invasive endovascular techniques (EVAR) available for prophylactic repair, ${ }^{1,2}$ the most important complications following aortic intervention remain cardiovascular events. These present a significant morbidity and mortality burden to patients with AAA. ${ }^{3,4}$ The reason for this high cardiovascular-risk status remains unclear but there is evidence that the infra-renal aortic diameter has prognostic significance, irrespective of a formal diagnosis of aneurysm (see Norman et al..$^{5}$ ).

The histopathological findings in AAA include inflammation, loss of vascular smooth muscle cells and disruption of elastin fibers, which are replaced by collagen, ${ }^{6,7}$ a mechanically stiffer substance. ${ }^{8}$ When aneurysm tissue is examined ex-vivo by biaxial biomechanical testing it exhibits increased stiffness and decreased distensibility compared with normal aorta. ${ }^{9}$ Tissue Doppler imaging measures of arterial stiffness within the AAA in-vivo corroborate this work. ${ }^{10}$ However, the data concerning any relationship between localized aortic stiffness and AAA growth/rupture are conflicting. ${ }^{11-14}$ These studies provide evidence of localized aortic stiffening within an aneurysm but give little insight into the status of the rest of the arterial tree in these patients. Interestingly, the Second Manifestations of Arterial Disease Study investigators reported increased stiffness at the carotid artery in patients with AAA, suggesting changes beyond the aneurysm itself. ${ }^{15}$

Generalized arterial stiffness is an independent predictor of cardiovascular morbidity and mortality and is elevated in end stage renal disease, ${ }^{16}$ diabetes ${ }^{17}$ and hypertension. ${ }^{18}$ Pulse wave velocity (PWV) is the speed at which the pulse wave travels through an arterial segment and provides a measure of arterial stiffness through the Moens-Korteweg equation. It can be measured non-invasively using one of a number of commercially available devices (reviewed in Davies et al. ${ }^{19}$ ). Current evidence reporting changes in PWV in patients with AAA is limited and conflicting, with relatively small sample sizes. ${ }^{20-22}$

${ }^{1}$ The Multidisciplinary Cardiovascular Research Centre, Leeds Institute of Genetics, Health \& Therapeutics, Division of Cardiovascular \& Diabetes Research, School of Medicine, University of Leeds, Leeds, UK; ${ }^{2}$ The Leeds Vascular Institute, The General Infirmary at Leeds, Leeds, UK and ${ }^{3}$ Leeds Institute of Genetics, Health \& Therapeutics, Division of Biostatistics, School of Medicine, University of Leeds, Leeds, UK

${ }^{4}$ These authors contributed equally to this work.

Correspondence: MA Bailey, BHF Clinical Research Fellow, The Leeds Vascular Institute, The General Infirmary at Leeds, Great George Street, Leeds LS1 3EX, UK. E-mail: marc.bailey@doctors.org.uk

Received 27 February 2014; revised 23 April 2014; accepted 1 May 2014; published online 12 June 2014 
In this study we set out to investigate the relationship between aortic diameter and the presence of AAA on PWV measured noninvasively using the Vicorder device.

\section{METHODS}

\section{Patients}

The study consisted of 309 patients from the same teaching hospital in the northern United Kingdom. Patients with known AAA were recruited from the vascular outpatients department while the control population were recruited from a range of surgical and medical outpatient departments of the same hospital on a voluntary basis between 1 January 2007 and 1 May 2013. At recruitment, all patients gave written, informed consent after receiving a patient information brochure and at least a $24 \mathrm{~h}$ cooling-off period. The study was given ethical approval by the local ethics committee (project reference: 03/142) and was conducted in accordance with the Declaration of Helsinki. After enrollment into the study all patients completed a detailed health questionnaire, administered face-to-face by a research nurse. This included medical history, concurrent medication use, smoking history and demographic data. An electrocardiogram was performed to identify any prior occult myocardial infarction.

\section{Aortic imaging and grouping}

The infra-renal abdominal aorta was imaged with B-mode ultrasound using a Titan SonoSite (SonoSite, Bothell, WA, USA). Maximal anterior-posterior aortic diameter was measured from inner wall to inner wall. Patients were split into AAA and control groups based on their aortic diameters, with an AAA being defined as an infra-renal abdominal aorta $\geqslant 3 \mathrm{~cm}$. Any patients recruited as controls who were subsequently found to have an incidental AAA were

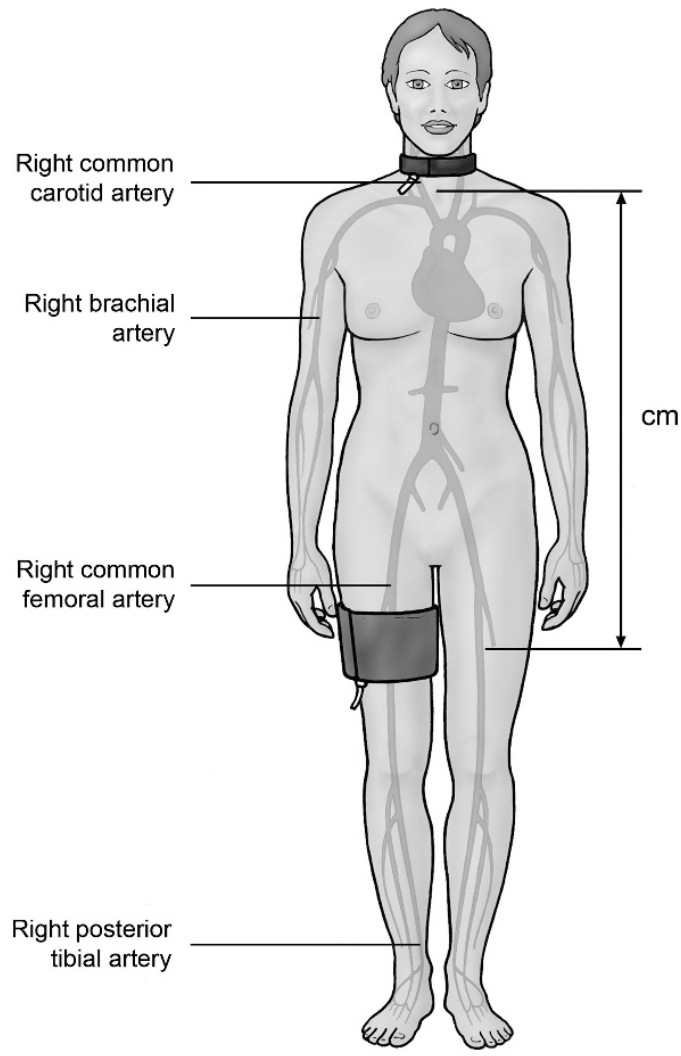

Figure 1 Cuff location and superficial distance measurements for Vicorder recording of carotid-femoral pulse wave velocity. The cuffs are positioned over the right common carotid artery and the right common femoral artery. The superficial distance is measured from the sternal notch to the middle of the femoral cuff (arrows) in centimeters $(\mathrm{cm})$. A full color version of this figure is available at the Hypertension Research journal online. transferred into the AAA group and referred to the vascular unit to receive routine care.

Blood samples, anthropometric and hemodynamic measurements All patients donated $50 \mathrm{mls}$ of free flowing venous blood from the antecubital vein of their non-dominant arm following an overnight fast, which was analyzed by the hospital laboratory for lipid screen (serum cholesterol, triglycerides, high density lipoprotein and low density lipoprotein). Height and weight were recorded using Statiometer and Column Scales, respectively, (Seca Medical Scales and Medical Systems, Birmingham, UK). Systolic (SBP) and diastolic blood pressure were recorded at the right brachial artery in duplicate using an Omtron M7 automated oscillometric device (Intelli sense, Kyoto, Japan); the mean of these repeats was used for analysis. The Ankle Brachial Pressure Index (ABPI) was calculated using the lowest pedal SBP in each foot divided by the highest brachial pressure as measured by a Dopplex 11 doppler probe (Huntleigh Diagnostics, Cardiff, UK) and a manual sphygmomanometer. The lowest ABPI was used in the later models.

\section{Pulse wave velocity}

Supine carotid-femoral PWV (cfPWV) measurements were determined by assessment with the Vicorder device (Skidmore Medical, Bristol, UK), a semiautomated, non-invasive system for determining cfPWV, which has been validated against the SphygmoCor device. ${ }^{23}$ The foot-to-foot transit time of each proximal and distal waveform was identified using the in-built crosscorrelation algorithm, enabling the computation of the time delay between the two. Simultaneous wave forms were observed to ensure there was no evidence of flow abnormality owing to atherosclerosis. Each measurement was made in duplicate with a $5 \mathrm{~min}$ rest period between measurements. For recordings a $30 \mathrm{~mm}$ pad was positioned over the neck at the level of the right carotid artery, with a larger $100 \mathrm{~mm}$ oscillometric cuff being positioned around the upper part of the right thigh. The path length was measured superficially with a tape measure, from the suprasternal notch to the middle of the femoral cuff, as per manufacturer's instructions (Figure 1). The heart rate of subjects was also measured during the $\mathrm{cfPWV}$ recording.

\section{Intra- and inter-observer variation}

A subset of 30 patients (15 AAA, 15 controls) was used to assess the variability of cfPWV. PWV measurements were performed twice by each observer in the same temperature controlled room. The cuffs were removed and re-applied between each measurement.

\section{Statistics}

Data were analyzed using SPSS v20 (SPSS. Chicago, IL, USA). Normality was assessed using the Shapiro-Wilk test and non-normal data log transformed to allow the use of parametric statistics. Continuous data is presented as mean \pm s.d. (unless stated) and categorical data as $n(\%) ; P$-values $<0.05$ were taken to be statistically significant.

Uni-variable comparisons were undertaken using student $t$-test or Fisher's exact test as appropriate. False discovery rate adjustment ${ }^{24}$ was employed to correct for multiple testing. Pearson's correlation coefficient was used to determine uni-variable parametric correlations between continuous variables and cfPWV. A binary logistic regression model was used to determine those covariables, which were independently statistically significantly different between AAA and control groups to guide inclusion in the model for cfPWV. Following this, a linear regression model was constructed to determine independent predictors of PWV, this model included factors, which remained significantly different between groups on binary logistic regression analysis, and factors which were correlated with cfPWV based both on the literature (for example, age, SBP) and bivariate correlation analysis. Observer variation was assessed using Bland and Altman analysis (limits of agreement) and the intraclass correlation coefficient.

\section{Power}

A sample size calculation for the pilot study (observer variation phase) was based on the standard deviation quoted in Hickson et al. ${ }^{23}$ To detect a 
Table 1 Baseline characteristics of the study population

\begin{tabular}{|c|c|c|c|c|c|}
\hline \multirow[b]{2}{*}{ Categorical variables } & \multicolumn{2}{|c|}{$\operatorname{Ctrl}(\mathrm{n}=161)$} & \multicolumn{2}{|c|}{$A A A(\mathrm{n}=148)$} & \multirow[b]{2}{*}{ P-value } \\
\hline & $n$ & $\%$ & $\mathrm{n}$ & $\%$ & \\
\hline Male sex & 120 & 74.5 & 128 & 86.4 & $0.009^{a}$ \\
\hline MI & 15 & 9.3 & 39 & 26.3 & 0.0002 \\
\hline Angina & 12 & 7.45 & 31 & 20.9 & $0.002^{\mathrm{a}}$ \\
\hline PVD & 13 & 8.0 & 41 & 27.7 & 0.0002 \\
\hline CVD & 20 & 12.4 & 20 & 13.5 & 0.52 \\
\hline DM & 19 & 11.8 & 28 & 18.9 & 0.11 \\
\hline HTN & 63 & 39.1 & 101 & 68.2 & $0.0002^{2}$ \\
\hline VTE & 5 & 3.1 & 8 & 5.4 & 0.64 \\
\hline Current smoker & 20 & 12.4 & 26 & 17.6 & 0.17 \\
\hline Ever smoker & 100 & 62.1 & 127 & 85.8 & $0.0002^{2}$ \\
\hline Aspirin & 54 & 33.5 & 114 & 77.0 & 0.0002 \\
\hline Statin & 73 & 45.3 & 116 & 78.3 & 0.0002 \\
\hline ACEi & 36 & 22.4 & 63 & 42.6 & 0.0002 \\
\hline BBs & 26 & 16.1 & 57 & 38.5 & $0.0002^{2}$ \\
\hline CCBs & 26 & 16.1 & 48 & 32.4 & $0.002^{a}$ \\
\hline Warfarin & 4 & 2.5 & 1 & 0.7 & 0.26 \\
\hline
\end{tabular}

\begin{tabular}{|c|c|c|c|c|c|}
\hline \multirow[b]{2}{*}{ Continuous variables } & \multicolumn{2}{|c|}{$\operatorname{Ctrl}(\mathrm{n}=161)$} & \multicolumn{2}{|c|}{$A A A(\mathrm{n}=148)$} & \multirow[b]{2}{*}{ P-value } \\
\hline & Mean & s.d. & Mean & s.d. & \\
\hline Aortic diameter $(\mathrm{cm})$ & 2.18 & 1.13 & 4.22 & 1.35 & $0.0002^{a}$ \\
\hline Age (years) & 69.7 & 7.8 & 73 & 7.5 & $0.0002^{\mathrm{a}}$ \\
\hline Pack years & 28.9 & 24.3 & 42.9 & 26.5 & $0.0002^{\mathrm{a}}$ \\
\hline Height (m) & 1.7 & 0.09 & 1.7 & 0.08 & 0.86 \\
\hline Weight (kg) & 80.7 & 14.1 & 81.3 & 16.2 & 0.79 \\
\hline BMI & 28 & 4.6 & 28 & 4.5 & 0.95 \\
\hline Pulse rate (BPM) & 66.3 & 11.5 & 68.4 & 13.8 & 0.16 \\
\hline Systolic BP (mm Hg) & 144.8 & 17.2 & 141.6 & 21.4 & 0.19 \\
\hline Diastolic BP (mm Hg) & 83 & 10.6 & 81.05 & 12.7 & 0.19 \\
\hline ABPI (Lowest) & 0.94 & 0.16 & 0.85 & 0.22 & $0.002^{\mathrm{a}}$ \\
\hline ABPI (Left) & 0.96 & 0.21 & 0.87 & 0.26 & 0.076 \\
\hline ABPI (Right) & 0.95 & 0.22 & 0.89 & 0.22 & 0.29 \\
\hline Cholesterol $\left(\mathrm{mmoll}^{-1}\right)$ & 4.7 & 1.3 & 4.2 & 0.9 & $0.0002^{\mathrm{a}}$ \\
\hline $\mathrm{TG}\left(\mathrm{mmol} \mathrm{I}^{-1}\right)$ & 1.39 & 0.85 & 0.68 & 1.53 & 0.069 \\
\hline $\mathrm{HDL}\left(\mathrm{mmol} \mathrm{I}^{-1}\right)$ & 1.43 & 0.46 & 1.21 & 0.37 & $0.0002^{\mathrm{a}}$ \\
\hline LDL (mmol $\left.\mathrm{I}^{-1}\right)$ & 2.79 & 1.07 & 2.41 & 1.36 & $0.01^{\mathrm{a}}$ \\
\hline
\end{tabular}

Abbreviations: $\mathrm{AAA}$, abdominal aortic aneurysm; $\mathrm{ABPI}$, ankle brachial pressure index $\mathrm{ACEi}$, angiotensin converting enzyme inhibitor; BBs, beta blockers; BMI, body mass index; $\mathrm{BP}$, blood pressure; BPM, beats per minute; CCBs, calcium channel blockers;

CVD, cerebrovascular disease; Ctrl, control; DM, diabetes mellitus; HDL, high density CVD, cerebrovascular disease; Ctrl, control; DM, diabetes mellitus; HDL, high density
lipoprotein; HTN, hypertension; LDL, low density lipoprotein; MI, myocardial infarction; PVD, peripheral vascular disease; TG, triglycerides; VTE, venous thromboembolism. a Statistically significant at $P<0.05$ level.

difference of $0.3 \mathrm{~ms}^{-1}$ between observers with $80 \%$ power and $95 \%$ confidence, a sample of 30 patients was required. Using the standard deviation data generated from this pilot study, a sample size calculation was undertaken for the main study. To detect a mean difference in PWV between groups of $1.0 \mathrm{~ms}^{-1}$ (which we felt was of clinical relevance as it equated to a $19 \%$ increase in cardiovascular mortality in a study using the Complior device ${ }^{25}$ ) with $80 \%$ power and $95 \%$ confidence, a sample of $\sim 300$ patients was required.

\section{RESULTS}

\section{Baseline characteristics}

Of the 309 patients included in the study, 148 had an aortic diameter $\geqslant 3 \mathrm{~cm}$ and formed the AAA group while the remaining 161 patients with aortic diameter $<3 \mathrm{~cm}$ formed the control group. The baseline characteristics of the study population are provided in Table 1, containing $P$-values based on uni-variable analysis with false discovery rate correction. Those co-variables that remained significant after false discovery rate correction (annotated with ${ }^{\mathrm{a}}$ ) were taken forward into a binary logistic regression model using the enter method $\left(R^{2}=0.73\right)$. In this multivariable model, the factors with statistically significant $P$ values were aortic diameter $(2.18 \pm 1.13 \mathrm{~cm}$ vs. $4.22 \pm 1.35 \mathrm{~cm}$, $P<0.001)$ and aspirin usage (33.5 vs. $77.0 \%, P=0.001)$. Age $(69.7$ years vs. 73.0 years, $P=0.06)$ and a prior history of myocardial infarction was of borderline significance $(9.3 \%$ vs. $26.4 \%, P=0.06)$. These factors were therefore taken forward as the most relevant potential confounders in the later linear model for cfPWV.

\section{Intra- and inter-observer variation in PWV measurements}

In the pilot study of 30 patients ( $15 \mathrm{AAA}$ and 15 controls) the mean difference for repeated cfPWV by a single observer was $-0.18 \mathrm{~ms}^{-1}$ with limits of agreement of -3.12 to $2.76 \mathrm{~ms}^{-1}$ and an intraclass correlation coefficient of $0.89(P<0.001)$. The mean difference for repeated cfPWV between observers was $0.11 \mathrm{~ms}^{-1}$ with limits of agreement of -3.41 to 3.63 . The intraclass correlation coefficient was $0.73(P<0.001)$.

\section{cfPWV for the study population}

The mean cfPWV for the entire study population was $9.65 \pm 2.3 \mathrm{~ms}^{-1}$, which is in keeping with the reference ranges published by the Arterial Stiffness Collaboration for patients aged 63years and over. ${ }^{26}$ In keeping with these data, there was a significant positive correlation between cfPWV and age (Figure 2a, $r=0.24, P<0.001$ ) and between cfPWV and SBP (Figure $2 b, r=0.29, P<0.001$ ). There was also a negative correlation between cfPWV and aortic diameter, which was both novel and highly statistically significant (Figure $2 c, r=-0.15, P=0.008$ ). Notably, there was no correlation between cfPWV and heart rate in our study population $(r=0.08, P=0.19)$ or $c f P W V$ and ABPI $(r=-0.023, P=0.7)$. These three significant correlations were taken forward into the linear regression model for cfPWV along with the three most relevant confounding variables from the binary logistic regression model (aortic diameter, aspirin usage and a prior history of myocardial infarction). A summary of bivariate correlation data is provided in Table 2.

\section{Comparison of cfPWV in AAA and control patients}

We could not identify any statistically significant difference in cfPWV between the control and AAA group: $9.75 \pm 2.3 \mathrm{~ms}^{-1} v s$. $9.55 \pm 2.3 \mathrm{~ms}^{-1}, P=0.43$ (Figure $3 \mathrm{a}$ ). When all relevant variables (AAA/control status, aortic diameter, age, SBP, aspirin use and a history of MI) were added into a simple linear regression model using the enter method with $\mathrm{cfPWV}$ as the dependent variable $\left(R^{2}=0.16\right.$, 6 degrees of freedom, $P<0.001)$, age $(\beta=0.24, P<0.001)$, SBP $(\beta=0.24, P<0.001)$ and aortic diameter $(\beta=-0.23, P=0.003)$ remained significant independent predictors of cfPWV. To further investigate this trend we compared those patients with large AAA (defined as $\geqslant 5 \mathrm{~cm}, n=32$ ) with those patients with a normal diameter aorta (defined as $<3 \mathrm{~cm}, n=161$ ) and the remaining patients with small AAA (defined as $3.0-4.9 \mathrm{~cm}, n=115$ ). In this analysis the large AAA group had decreased cfPWV as compared with the control group, mean difference: $1.07 \mathrm{~ms}^{-1}(95 \%$ confidence interval of the mean difference: $0.2 \mathrm{~ms}^{-1}$ to $1.9 \mathrm{~ms}^{-1}, P=0.02$ ) and patients with small AAA mean difference: $1.11 \mathrm{~ms}^{-1}(95 \%$ confidence interval of the mean difference: $2.0 \mathrm{~ms}^{-1}$ to $0.2 \mathrm{~ms}^{-1}$, $P=0.02$ ) (Figure $3 \mathrm{~b}$ ). 

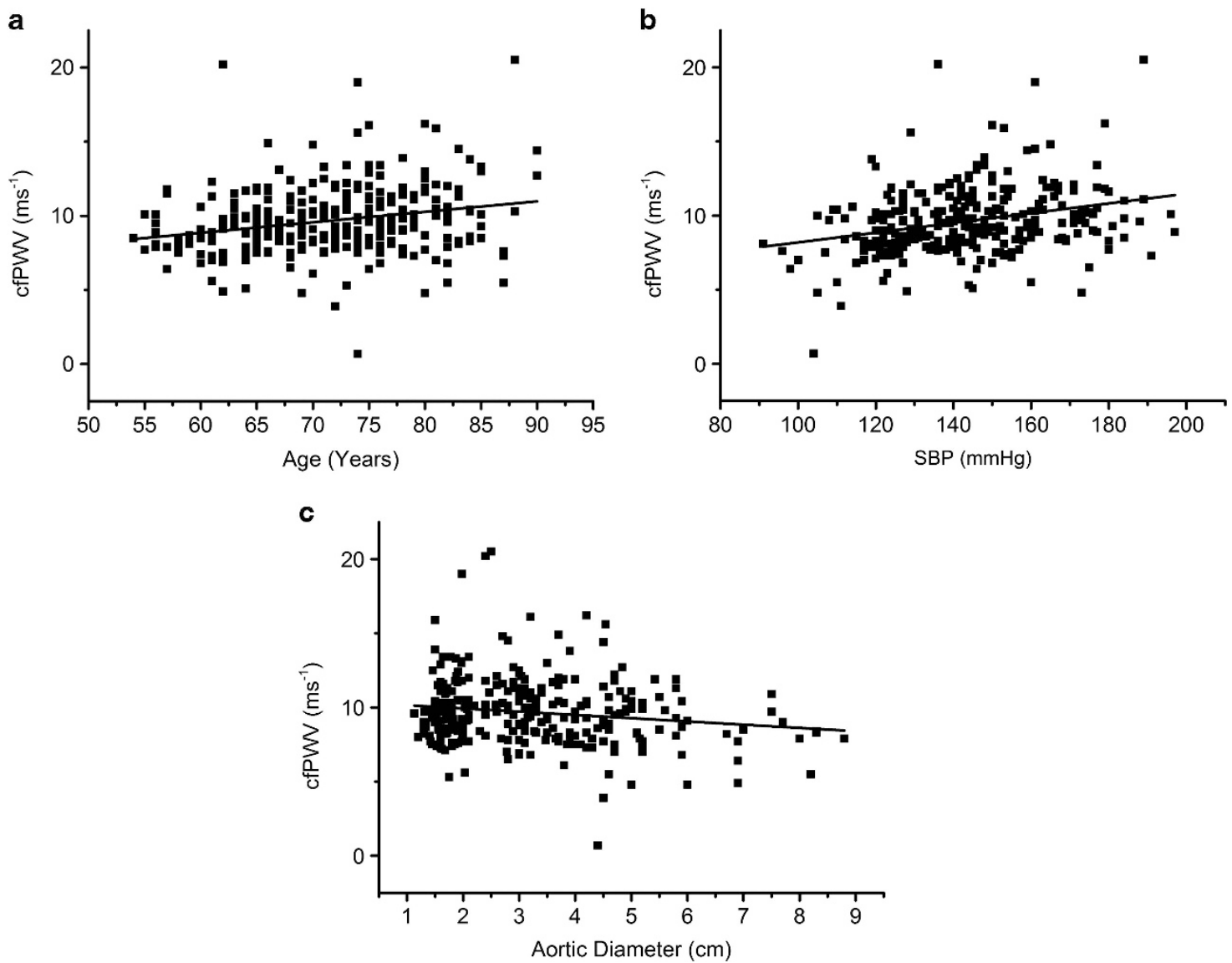

Figure 2 Scatterplots demonstrating significant correlations with carotid-femoral pulse wave velocity (cfPWV). (a) Significant positive correlation between age and cfPWV, $r=0.244, P<0.001$. (b) Significant positive correlation between systolic blood pressure (SBP) and $c f P W V, r=0.29, P<0.001$. (c) Significant negative correlation between aortic diameter and cfPWV, $r=-0.15, P=0.008$.

Table 2 Bivariate correlation analysis of cfPWV with continuous variables of interest

\begin{tabular}{|c|c|c|}
\hline Pearson's correlation vs. cfPWV & Pearson's R & P-value \\
\hline Aortic diameter $(\mathrm{cm})$ & -0.15 & $0.008^{a}$ \\
\hline Age (years) & 0.24 & $<0.001^{a}$ \\
\hline Pack years & -0.44 & 0.57 \\
\hline Height (m) & 0.02 & 0.73 \\
\hline Weight (kg) & -0.15 & 0.79 \\
\hline BMI & -0.22 & 0.69 \\
\hline Systolic BP (mm Hg) & 0.29 & $<0.001^{a}$ \\
\hline Diastolic BP (mm Hg) & 0.08 & 0.14 \\
\hline ABPI (lowest) & -0.023 & 0.69 \\
\hline ABPI (left) & -0.026 & 0.66 \\
\hline ABPI (right) & -0.026 & 0.65 \\
\hline Cholesterol $\left(\mathrm{mmoll}^{-1}\right)$ & -0.31 & 0.59 \\
\hline $\mathrm{TG}(\mathrm{mmoll}-1)$ & -0.009 & 0.88 \\
\hline $\mathrm{HDL}\left(\mathrm{mmoll}^{-1}\right)$ & 0.07 & 0.23 \\
\hline $\mathrm{LDL}\left(\mathrm{mmol}^{-1}\right)$ & -0.04 & 0.47 \\
\hline
\end{tabular}

Abbreviations: $\mathrm{ABPI}$, ankle brachial pressure index; $\mathrm{BMI}$, body mass index; $\mathrm{BP}$, blood pressure; HDL, high density lipoprotein; LDL, low density lipoprotein; TG, triglycerides.

a Statistically significant at $P<0.05$ level.

\section{DISCUSSION}

In the present study we investigated arterial stiffness in a cohort of patients with and without AAA using cfPWV as measured by the
Vicorder device. We demonstrated high repeatability of the measurement technique and included a sample size with enough power to detect a $1.0 \mathrm{~ms}^{-1}$ difference in PWV between groups. Our variability data was in keeping with another recent study in patients with peripheral arterial disease using the same device. ${ }^{27}$ We found no difference in cfPWV between the AAA and control groups; however, a small but significant negative correlation was observed between aortic size and cfPWV. Further, patients with the largest aneurysms in the study $(\geqslant 5 \mathrm{~cm})$ had lower cfPWV than controls and patients with small AAA $(<5 \mathrm{~cm})$.

It is well-established that increasing PWV occurs with age and hypertension, ${ }^{18,26}$ is associated with a worsening cardiovascular-risk profile $^{28}$ and can be elevated with increased heart rate. ${ }^{29} \mathrm{PWV}$ is appealing as it represents one of the only non-invasive methods to detect changes within the vascular tree. It is of interest with regards to AAA owing to the unexplained increased cardiovascular morbidity and mortality observed after intervention ${ }^{3,4}$ and evidence suggesting high arterial stiffness within an AAA. ${ }^{9,10}$

The PWV provides a global estimate of arterial stiffness as described by the Moens-Korteweg equation $(\mathrm{PWV}=\sqrt{ }[E h / 2 r \rho])$, which states that PWV is proportional to the square root of the elastic modulus $(E)$ of the vessel wall given a constant ratio of wall thickness $(h)$ to vessel radius $(r)$ and blood density $(\rho)$ assuming isotropy. It is this type of stiffness estimation that is most strongly linked to the cardiovascular-risk profile. Few groups have investigated differences in arterial stiffness as measured by PWV in patients with AAA (and a 

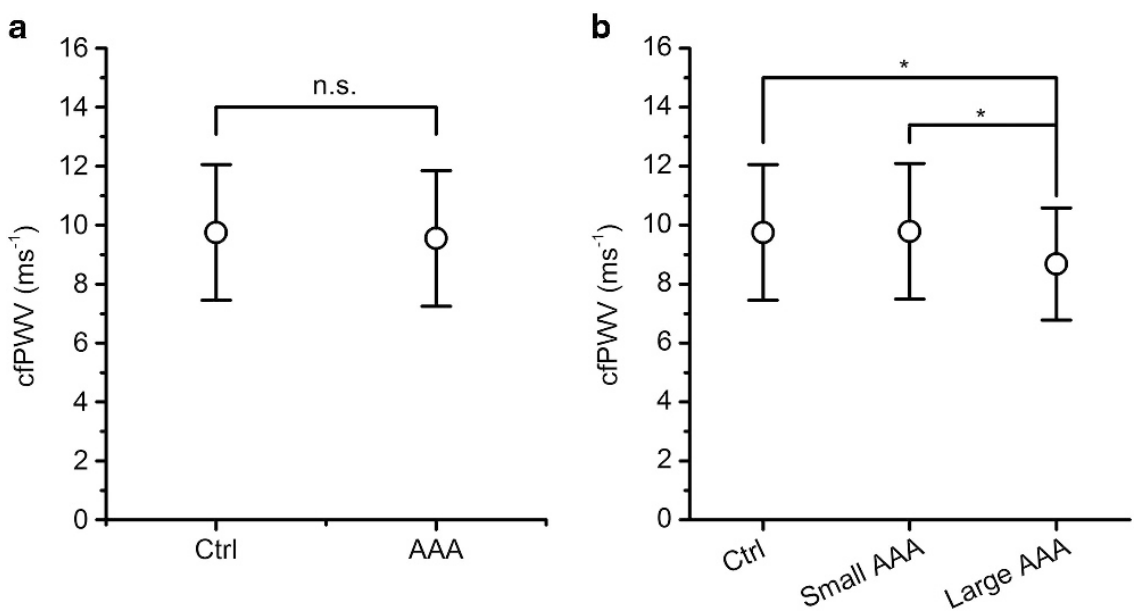

Figure 3 Comparison of carotid-femoral pulse wave velocity (cfPWV) in AAA and control patients. (a) No significant difference between control and AAA patients for cfPWV. (b) Significantly lower cfPWV values in patients with large AAA compared with small AAA $(P=0.02)$ and control patients $(P=0.02)$; ${ }^{*} P<0.05$. n.s., not significant.

suitable control population). Currently there is equipoise in the literature. Kadoglou et al. ${ }^{20,21}$ reported increased cfPWV $\left(12.99 \pm 3.75 \mathrm{~ms}^{-1}\right.$ vs. $\left.10.03 \pm 1.57 \mathrm{~ms}^{-1}, P<0.001\right)$ in 108 patients with AAA compared with 41 age- and sex-matched controls with angiographically proven coronary artery disease, using the Complior device. Conversely, Lee et al. ${ }^{22}$ reported a lower cfPWV in 51 AAA patients compared with 51 age-, sex- and SBP-matched controls $\left(12.1 \pm 2.7 \mathrm{~ms}^{-1} \quad\right.$ vs. $\left.\quad 13.6 \pm 3.5 \mathrm{~ms}^{-1}, \quad P=0.009\right) \quad$ recruited as individuals participating in other hemodynamic studies, using the Collins VP-2000 device (Wave Nexus Corp., San Antonio, TX, USA). However, in a murine model system of AAA, no difference in PWV was found between angiotensin II infused (aneurysmal) and sham operated animals. ${ }^{30}$

Given the higher atherosclerotic disease burden in our aneurysm group the finding of a lower cfPWV in the patients with the largest AAAs was surprising. One possible explanation for this may have been a difference in heart rates between the two groups owing to the differences observed in beta blockade. A lower heart rate in the AAA group would be expected to reduce the cfPWV. ${ }^{29}$ However, when we studied heart rate, it was similar in both groups and further, was not significantly correlated with $\mathrm{cfPWV}$ in the whole study population. Our findings support those of Lee et al. ${ }^{22}$ and we provide a similar explanation. The Moens-Korteweg equation presumes isotropy within the arterial segment measured, which is not the case in AAA, where the vessel is, by definition, thinned and anisotropic. ${ }^{9}$ This invalidates the assumptions of the Moens-Korteweg equation and in this situation, reduced cfPWV may simply be a surrogate marker of a larger aortic diameter. This is reinforced by reports that PWV increases after deployment of an aortic stent graft. $^{20}$ The deployment of an aortic stent graft restores isotropy to the aorta and a reversal of any decrease in cfPWV relating to aortic diameter would therefore be expected. In our study cfPWV was correlated with aortic size in all patients and was lowest in patients with the largest aneurysms $v s$. controls but not when the whole AAA and control group were compared. This is likely owing to the small difference in means between both groups $\left(0.21 \mathrm{~ms}^{-1}\right)$, which lay well below $1.0 \mathrm{~ms}^{-1}$ that to which the study was powered to detect. To detect a difference of this magnitude with statistical significance given the variability we observed, a sample of some 4150 patients would be required. Further, it is difficult to know if this difference would be of any clinical significance. Our results, along with those of Lee et al. ${ }^{22}$ suggest strongly that cfPWV is unlikely to provide an accurate measurement of arterial stiffness in patients with AAA owing to the apparent confounding effect of aortic diameter. It could be argued that a lower than expected (for age and SBP) cfPWV may be a warning sign of AAA presence. However, given the patient-to-patient variability in cfPWV observed in this and other studies and the fact that we observed no significant difference between AAA and control groups, it seems unlikely that this approach would be sensitive enough for use as a viable screening tool. Of potentially more interest, is the reported change in cfPWV after endovascular techniques. Further work is required in this area, but in the context of our results it would be valuable to determine if a decrease in cfPWV after stent graft insertion could be used to detect sac expansion and the 'at-risk aortic endoprosthesis' and thus reduce the burden of aortic imaging required in these patients. ${ }^{31}$ Further work should be directed towards methods, which can determine aortic stiffness both within and away from the aneurysm itself. Such regional PWV measures can be achieved accurately with magnetic resonance imaging, for example.

There are notable differences between the study designs in the three studies that report cfPWV measures in AAA compared with a control group, which may influence the cfPWV data reported. All three studies used different devices to measure cfPWV. We chose the Vicorder as it has been validated against the SphygmoCor device, ${ }^{23}$ which is simple to use and results are repeatable between users, as confirmed by our data and other studies. ${ }^{23,27}$ Reassuringly, we observed a significant positive correlation between Vicorder measured cfPWV, age and SBP, in keeping with previous pulications. ${ }^{18,26}$ The Complior device is well-established and has proven validity and consistency. ${ }^{19}$ However, The VP-2000 device has not been validated for PWV; only validation for ABPI measurement has been published for this device. ${ }^{32}$ The patient populations originate from diverse geographical locations (UK, Greece, Taiwan) and the recruitment strategy is different. Our cohort of AAA patients had smaller aneurysms than those in either the Kadoglou et al. ${ }^{20,21}$ or Lee et al. ${ }^{22}$ studies $(4.2 \mathrm{~cm}$ vs. $6.3 \mathrm{~cm}$ and $5.4 \mathrm{~cm}$, respectively), which may blunt a size-dependent effect. Further, while we chose to recruit a control group from other hospital outpatient clinics, Kadoglou et al. ${ }^{21}$ only used patients with confirmed coronary artery disease, which one 
would expect to impact arterial stiffness ${ }^{33-35}$ while Lee et al. ${ }^{22}$ provided limited information on their control recruitment strategy, saying only that controls were 'selected from individuals participating in other hemodynamic studies.'

Despite the comparatively large sample size there are a number of important limitations to our study. First, owing to our study design our control group was not age- or sex-matched to the AAA group and our AAA group had a higher burden of atherosclerotic disease. Despite using multivariable statistical approaches to attempt to control for confounding factors it is impossible to completely eliminate the differences between our two patient cohorts. We also included some 54 patients with peripheral arterial disease, which could impact on PWV measurement by the Vicorder device, as a proximal atherosclerotic lesion would delay the waveform transit to the femoral cuff. However, we did not observe any evidence of flow abnormality based on simultaneous waveform analysis, none of our patients had aortic occlusive disease and we did not identify any correlation between ABPI (lowest, right or left) and cfPWV. The determination of path length for cfPWV determination is also relatively inexact, requiring superficial distance measurement with a tape measure. There are differences between true magnetic resonance imaging path length and superficial distance measures, which can be corrected mathematically. ${ }^{36}$ However, AAA is associated with arterial tortuosity, ${ }^{37}$ which suggests distance measurement could only be improved by additional imaging. This issue could also be resolved by using magnetic resonance imaging. There is also the possibility of type II error when comparing overall AAA and control groups for cfPWV owing to the magnitude of the difference being below that to which the study was powered but above the observer related error we observed $\left(0.11 \mathrm{~ms}^{-1}\right)$. Finally, we only measured cfPWV at a single time interval in the patient groups. Future studies should use a longitudinal study design to investigate changes in cfPWV as the aneurysm progresses. Although a much larger sample of patients would be required for this analysis than used in the present study in order to perform sufficient aneurysm growth modeling. ${ }^{38}$

In conclusion, the Vicorder is a simple non-invasive device, which produces repeatable estimates of cfPWV. We demonstrated a significant negative correlation between cfPWV and infra-renal aortic diameter and reduced cfPWV in patients with large AAA compared with patients with either small AAA or normal aortas despite the high atherosclerotic burden in this group. This suggests cfPWV is a potentially invalid method for accurately determining arterial stiffness in patients with AAA owing to the confounding effects of aortic size.

\section{CONFLICT OF INTEREST}

The authors declare no conflict of interest.

\section{ACKNOWLEDGEMENTS}

We would like to thank Janet Woods for data collection, David Watson for data inputting, Thomas Flemming for database programming and management, Richard Cubbon for critiquing the manuscript and Paul Brown for producing the line drawing. Funding for the study was received from the Garfield Weston Trust for Research into Heart Surgery and the Special Trustees of the Leeds Teaching Hospitals NHS Trust. MAB, KJG and SS have received personal funding from the National Institute for Health Research. MAB, KJG and KIB have received personal funding from the British Heart Foundation. Pilot data from this work was presented at the UK Medical Students Conference, 2011 and the Association of Surgeons of Great Britain and Ireland International Meeting, 2012. The authors confirm there has been no duplicate submission or prior publication.
1 Chambers D, Epstein D, Walker S, Fayter D, Paton F, Wright K, Michaels J, Thomas S, Sculpher M, Woolacott N. Endovascular stents for abdominal aortic aneurysms: a systematic review and economic model. Health Technol Assess 2009; 13: 215-318.

2 Filardo G, Powell JT, Martinez MA, Ballard DJ. Surgery for small asymptomatic abdominal aortic aneurysms. Cochrane Database Syst Rev 2012; 3: CD001835.

3 Brown LC, Thompson SG, Greenhalgh RM, Powell JT, Endovascular Aneurysm Repair trial participants. Incidence of cardiovascular events and death after open or endovascular repair of abdominal aortic aneurysm in the randomized evar trial 1 . Br J Surg 2011; 98: 935-942.

4 Karthikesalingam A, Bahia SS, Patterson BO, Peach G, Vidal-Diez A, Ray KK, Sharma R, Hinchliffe RJ, Holt PJ, Thompson MM. The shortfall in long-term survival of patients with repaired thoracic or abdominal aortic aneurysms: Retrospective case-control analysis of hospital episode statistics. Eur J Vasc Endovasc Surg 2013; 46: 533-541.

5 Norman PE, Muller J, Golledge J. The cardiovascular and prognostic significance of the infrarenal aortic diameter. J Vasc Surg 2011; 54: 1817-1820.

$6 \mathrm{He}$ CM, Roach MR. The composition and mechanical properties of abdominal aortic aneurysms. J Vasc Surg 1994; 20: 6-13.

7 Jagadesham VP, Scott DJ, Carding SR. Abdominal aortic aneurysms: An autoimmune disease? Trends Mol Med 2008; 14: 522-529.

8 Dobrin PB, Baker WH, Gley WC. Elastolytic and collagenolytic studies of arteries. Implications for the mechanical properties of aneurysms. Arch Surg 1984; 119: 405-409.

9 Vande Geest JP, Sacks MS, Vorp DA. The effects of aneurysm on the biaxial mechanical behavior of human abdominal aorta. J Biomech 2006; 39: 1324-1334.

10 MacSweeney ST, Young G, Greenhalgh RM, Powell JT. Mechanical properties of the aneurysmal aorta. Br J Surg 1992; 79: 1281-1284.

11 Hoegh A, Lindholt JS. Basic science review. Vascular distensibility as a predictive tool in the management of small asymptomatic abdominal aortic aneurysms. Vasc Endovascular Surg 2009; 43: 333-338.

12 Long A, Rouet L, Vitry F, Albertini JN, Marcus C, Clement C. Compliance of abdominal aortic aneurysms before and after stenting with tissue doppler imaging: Evolution during follow-up and correlation with aneurysm diameter. Ann Vasc Surg 2009; 23: 49-59.

13 Ruegg G, Mason RH, Hardinge M, Perkins J, Husmann M, Russi EW, Bloch KE, Stradling JR, Kohler M. Augmentation index and central aortic blood pressure in patients with abdominal aortic aneurysms. J Hypertens 2010; 28: 2252-2257.

14 Wilson KA, Lee AJ, Lee AJ, Hoskins PR, Fowkes FG, Ruckley CV, Bradbury AW. The relationship between aortic wall distensibility and rupture of infrarenal abdominal aortic aneurysm. J Vasc Surg 2003; 37: 112-117.

15 Dijk JM, van der Graaf Y, Grobbee DE, Banga JD, Bots ML, Group SS. Increased arterial stiffness is independently related to cerebrovascular disease and aneurysms of the abdominal aorta: the second manifestations of arterial disease (smart) study. Stroke 2004; 35: 1642-1646.

16 Blacher J, Safar ME, Guerin AP, Pannier B, Marchais SJ, London GM. Aortic pulse wave velocity index and mortality in end-stage renal disease. Kidney Int 2003; 63: $1852-1860$.

17 Woolam GL, Schnur PL, Vallbona C, Hoff HE. The pulse wave velocity as an early indicator of atherosclerosis in diabetic subjects. Circulation 1962; 25: 533-539.

18 Laurent S, Boutouyrie P, Asmar R, Gautier I, Laloux B, Guize L, Ducimetiere P, Benetos A. Aortic stiffness is an independent predictor of all-cause and cardiovascular mortality in hypertensive patients. Hypertension 2001; 37: 1236-1241.

19 Davies JM, Bailey MA, Griffin KJ, Scott DJ. Pulse wave velocity and the non-invasive methods used to assess it: Complior, sphygmocor, arteriograph and vicorder. Vascular 2012; 20: 342-349.

20 Kadoglou NP, Moulakakis KG, Papadakis I, Ikonomidis I, Alepaki M, Lekakis J, Liapis CD. Changes in aortic pulse wave velocity of patients undergoing endovascular repair of abdominal aortic aneurysms. J Endovasc Ther 2012; 19: 661-666.

21 Kadoglou NP, Papadakis I, Moulakakis KG, Ikonomidis I, Alepaki M, Moustardas P, Lampropoulos S, Karakitsos P, Lekakis J, Liapis CD. Arterial stiffness and novel biomarkers in patients with abdominal aortic aneurysms. Regul Pept 2012; 179: 50-54.

22 Lee CW, Sung SH, Chen CK, Chen IM, Cheng HM, Yu WC, Shih CC, Chen CH. Measures of carotid-femoral pulse wave velocity and augmentation index are not reliable in patients with abdominal aortic aneurysm. J Hypertens 2013; 31: 1853-1860.

23 Hickson SS, Butlin M, Broad J, Avolio AP, Wilkinson IB, McEniery CM. Validity and repeatability of the vicorder apparatus: a comparison with the sphygmocor device. Hypertens Res 2009; 32: 1079-1085.

24 Benjamini Y, Hochberg Y. Controlling the false discovery rate: A practical and powerful approach to multiple testing. J R Stat Soc Ser B Stat Methodol 1995; 57: 289-300.

25 Meaume S, Benetos A, Henry OF, Rudnichi A, Safar ME. Aortic pulse wave velocity predicts cardiovascular mortality in subjects $>70$ years of age. Arterioscler Thromb Vasc Biol 2001; 21: 2046-2050.

26 Reference Values for Arterial Stiffness Colaboration. Determinants of pulse wave velocity in healthy people and in the presence of cardiovascular risk factors: 'Establishing normal and reference values'. Eur Heart J 2010; 31: 2338-2350.

27 Shahin Y, Barakat H, Barnes R, Chetter I. The vicorder device compared with sphygmocor in the assessment of carotid-femoral pulse wave velocity in patients with peripheral arterial disease. Hypertens Res 2013; 36: 208-212.

28 Mattace-Raso FU, van der Cammen TJ, Hofman A, van Popele NM, Bos ML, Schalekamp MA, Asmar R, Reneman RS, Hoeks AP, Breteler MM, Witteman JC. 
Arterial stiffness and risk of coronary heart disease and stroke: the rotterdam study. Circulation 2006; 113: 657-663.

29 Millasseau SC, Stewart AD, Patel SJ, Redwood SR, Chowienczyk PJ. Evaluation of carotid-femoral pulse wave velocity: influence of timing algorithm and heart rate. Hypertension 2005; 45: 222-226.

30 Luo J, Fujikura K, Tyrie LS, Tilson MD, Konofagou EE. Pulse wave imaging of normal and aneurysmal abdominal aortas in vivo. IEEE Trans Med Imaging 2009; 28: 477-486.

31 Propper BW, Abularrage CJ. Long-term safety and efficacy of endovascular abdominal aortic aneurysm repair. Vasc Health Risk Manag 2013; 9: 135-141.

32 Takahashi I, Furukawa K, Ohishi W, Takahashi T, Matsumoto M, Fujiwara S. Comparison between oscillometric- and doppler-abi in elderly individuals. Vasc Health Risk Manag 2013; 9: 89-94.

33 Ben-Shlomo Y, Spears M, Boustred C, May M, Anderson SG, Benjamin EJ, Boutouyrie $\mathrm{P}$, Cameron J, Chen $\mathrm{CH}$, Cruickshank JK, Hwang SJ, Lakatta EG, Laurent S, Maldonado J, Mitchell GF, Najjar SS, Newman AB, Ohishi M, Pannier B, Pereira T, Vasan RS, Shokawa T, Sutton-Tyrell K, Verbeke F, Wang KL, Webb DJ, Hansen TW, Zoungas S, McEniery CM, Cockcroft JR, Wilkinson IB. Aortic pulse wave velocity improves cardiovascular event prediction: an individual participant meta-analysis of prospective observational data from 17,635 subjects. J Am Coll Cardiol 2013; 63: 636-646.

34 Cho SW, Kim BK, Kim JH, Byun YS, Goh CW, Rhee KJ, Ahn HS, Lee BK, Kim BO. Non-invasively measured aortic wave reflection and pulse pressure amplification are related to the severity of coronary artery disease. J Cardiol 2013; 62: 131-137.

35 Gaszner B, Lenkey Z, Illyes M, Sarszegi Z, Horvath IG, Magyari B, Molnar F, Konyi A, Cziraki A. Comparison of aortic and carotid arterial stiffness parameters in patients with verified coronary artery disease. Clin Cardiol 2012; 35: 26-31.

36 Huybrechts SA, Devos DG, Vermeersch SJ, Mahieu D, Achten E, de Backer TL, Segers P, van Bortel LM. Carotid to femoral pulse wave velocity: A comparison of real travelled aortic path lengths determined by MRI and superficial measurements. J Hypertens 2011; 29: 1577-1582.

37 Pappu S, Dardik A, Tagare H, Gusberg RJ. Beyond fusiform and saccular: A novel quantitative tortuosity index may help classify aneurysm shape and predict aneurysm rupture potential. Ann Vasc Surg 2008; 22: 88-97.

38 Bailey MA, Baxter PD, Jiang T, Charnell AM, Griffin KJ, Johnson A, Bridge KI, Sohrabi S, Scott DJ. Modeling the growth of infra-renal abdominal aortic aneurysms. Aorta 2013; 1: 258-273. 